An. Med InTERNa (Madrid) Vol. 18, N. ${ }^{\circ}$ 4, pp. 201-204, 2001

\title{
Embolismo por cristales de colesterol. A propósito de un caso
}

\author{
A. SANTAMARÍA, A. PERELLÓ, A. BERENGUER, F.J. VERA-SEMPERE*, J.R. \\ CALABUIG
}

Servicio de Medicina Interna y *Anatomía Patológica. Hospital La Fe. Valencia.

\author{
CHOLESTEROL CRYSTALS EMBOLIZATION. CASE REPORT
}

\section{RESUMEN}

El embolismo por cristales de colesterol es una entidad poco frecuente que se da en personas con enfermedad arteroesclerótica de forma espontánea o tras la realización de manipulación vascular (cateterismos arteriales, arteriografías, cirugía cardiovascular...) o más raramente con el tratamiento con anticuagulantes orales y/o heparina o tras fibrinolisis.

Da una variada sintomatología según el territorio arterial afecto y así existen desde formas diagnosticadas en necropsias hasta formas de clínica florida. Las más frecuentes son las manifestaciones cutáneas con aparición de livedo reticularis y/o dedos púrpuras que pueden ir acompañado de necrosis de miembros inferiores, seguida de la afectación renal y de la gastrointestinal. El diagnóstico se basa en la visualización de cristales de colesterol en biopsia del órgano afecto a nivel de arteriolas, sobre todo la biopsia cutánea, muscular y en algún caso renal. En cuanto al tratamiento es sintomático con analgésicos, antiagregantes plaquetarios, antiinflamatorios, etc., pero el curso del proceso es independiente del aplicado y no cambia la evolución del mismo. Existe una clara contraindicación al uso de anticuagulantes orales y heparina.

PALABRAS CLAVE: Émbolos de colesterol. Ateroesclerosis. Embolización. Colesterol.

\begin{abstract}
Cholesterol Crytals Embolization is not a very frequent entity that happens in people with atheromatous disease of spontaneus for or after the realization of vascular manipulation (angiographic, angioplaty pro cedures, cardiovascular surgery) or more rarely with the treatment with oral anticuagulants and after trombolytic therapy. The clinical presenta tion is according to the arterial affected territory and so from diagnosed cases in autopsy reports to florid clinic forms exist. The more frequent are the cutaneus manifestations with apparition of livedo reticularis and purple toes that could go accompanied of necrosis of lower limbs, conse cutive from the renal manifestations and the gastrointestinal trac one. The diagnosis is based on the biopsy of organ affected lesons to level of arterioles, that revealed characteristic cholesterol clefts, above all the cutaneus biopsy, muscular and in any case renal.

As for the treatment is symptomatic with analgesics, antiplatelet agents, antiinflammatory, etc... but it doesn't after the presage and the evolution of the illness. A clear contraindication exists to the use of oral anticuagulants and heparin.
\end{abstract}

KEY WORDS: Cholesterol embolic. Atheromatous. Embolization. Cho lesterol.

Santamaría A, Perelló A, Berenguer A, Vera-Sempere F.J, Calabuig J.R. Embolismo por cristales de colesterol. An Med Interna (Madrid) 2001; 18: 201-204

\section{INTRODUCCIÓN}

La enfermedad por embolias de cristales de colesterol (EECC) es una entidad anatomoclínica que se presenta en personas con enfermedad aterosclerótica avanzada de forma espontánea o tras manipulación arterial instrumental diagnóstica y/o terapéutica. Se presenta con variada sintomatología desde formas que pasan desapercibidas y son hallazgos causales necrópsicos hasta casos con clínica florida y diferentes niveles de gravedad. Debido a esa dificultad diagnóstica creemos interesante presentar un caso clínico muy ilustrativo de la entidad que nos sirve para recordar brevemente en que consiste y revisar los avances terapéuticos y sobre todo intentar que esté en la mente de cualquier clínico cuando se plantee un diagnóstico diferencial.

\section{CASO APORTADO}

Varón de 44 años, con hábito tabáquico, hipercolesterolemia tratada de forma irregular con dieta e hipocolesterolemiantes, y cardiopatía isquémica crónica (con infarto de miocardio diafragmático en 1985). Ingresa para estudio angiográfico por aparición de crisis de angor, tanto de esfuerzo como de reposo, en el último año a pesar del tratamiento con B-bloqueantes, nitratos, antiagregantes plaquetarios, calcioantagonistas, etc. Tras la realización del cateterismo arterial y coronariografía sin incidencias (se constató Enfermedad de Tres Vasos) fue remitido a su domicilio a las 24 horas y 12 horas después inicia un cuadro de disestesias con dolor a nivel de ambas pantorrillas que fue incrementando en los dos días siguientes por lo que acudió al servicio de urgencias del hospital y fue remitido a su domicilio con medicación analgésica. Acude nuevamente a dicho servicio 24 horas después por aparición de múltiples lesiones eritematosas en la piel de la mitad inferior del abdomen y en extremidades inferiores. Ingresa para estudio.

Exploración física: Varón, 44 años, normolineo; afebril; buena

Trabajo aceptado: 19 Junio de 1999

Correspondencia: A. Santamaría. Avd. Constitución, 126 - 7. 46009 Valencia. 


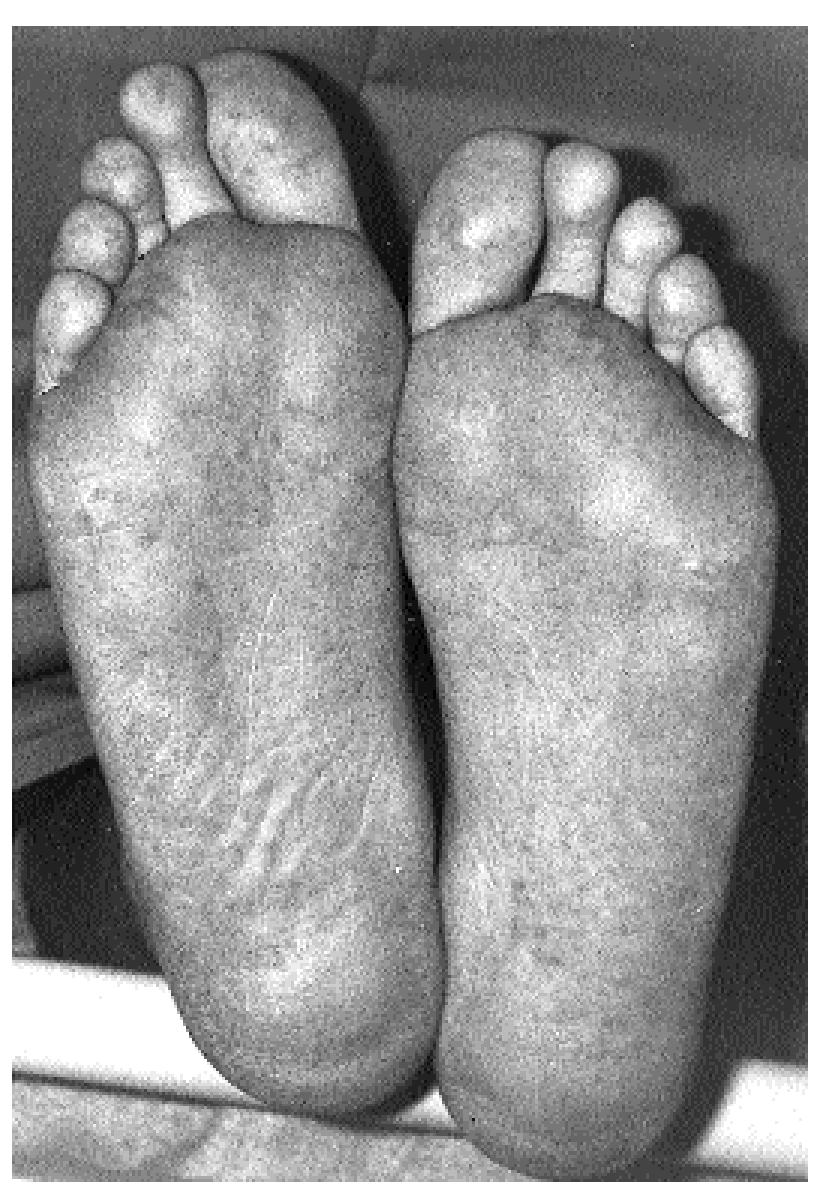

Fig. 1. Pequeños elementos maculares purpúricos configurando una imagen de livedo reticularis de carácter bilateral a nivel plantar.

coloración de piel y mucosas; xantelasma bilateral. PA:105/65. ACP: Frecuencia central rítmica a $62 \mathrm{ppm}$. Abdomen: Blando, indoloro a la palpación, sin visceromegalias ni masas. Extremidades sin edemas, con masas gemelares dolorosas a la palpación. Pulsos femoral, tibial posterior y pedio palpables y siméticos. A nivel cutáneo (en mitad inferior de abdomen y extremidades inferiores) se aprecian lesiones eritematosas compatibles con livedo reticularis y en dedos de los pies coloración púrpura con temperatura local normal.

Pruebas complementarias. Hemograma: Leucocitos 10800 (Seg 43\%, L 36\%, M 6,1\%, Eosinófilos 13\%) Hgb: 13,9 g/dl, Hct: 42,7\%, Plaquetas 203.000. VSG: 16. Bioquímica: Glu $129 \mathrm{mg} / \mathrm{dl}$, Urea $30 \mathrm{mg} / \mathrm{dl}$, Cre 1,1 mg/dl, Na $138 \mathrm{mEq} / \mathrm{l}, \mathrm{K}$ 4,4 mEq/l, GOT 25, GPT 31, CK 50, LDH 337, Aldolasa <8 U/l, Colesterol total 232, HDL 28, LDL 168, TG 179, APO B/ APO A1: >1.51. Hemostasia: Quick 100\%, Fibrinógeno 335. Orina: 25 leucos/ campo. PTG: Prot total 6,3 g/l, Alb 64\%, Alfa 1-G 3,5\%, Alfa 2-G 9,8\%, Beta-G 11,2\%, Gamma-G 12,8\%. Complemento: C3 111 (83-177 $\mathrm{mg} / \mathrm{dl})$, C4 27(15-45). Detección de anticuerpos antitejido: Negativa. Complejos inmunocirculantes: Positivos para Ig M. Radiografía tórax/abdomen: Sin hallazgos. Eco-Doppler MMII: Venas ilíacas, femorales y poplíteas permeables, sin signos de trombosis y con flujo normal. ECG: RS a 62 ppm, BCRDHH, necrosis de cara inferior residual. Examen oftalmológico: Normal. EMG (realizado a los 21 días): Se apreció lesión radicular irritativa en evolución S1 derecha leve.

Se realizó una biopsia cutánea de cara lateral de pie derecho que reveló la presencia a nivel de pequeñas arteriolas de la dermis media y profunda de cristales de colesterol enclavado intraluminalmente con presencia de ocasionales células gigantes fagocitando en parte estos cristales.

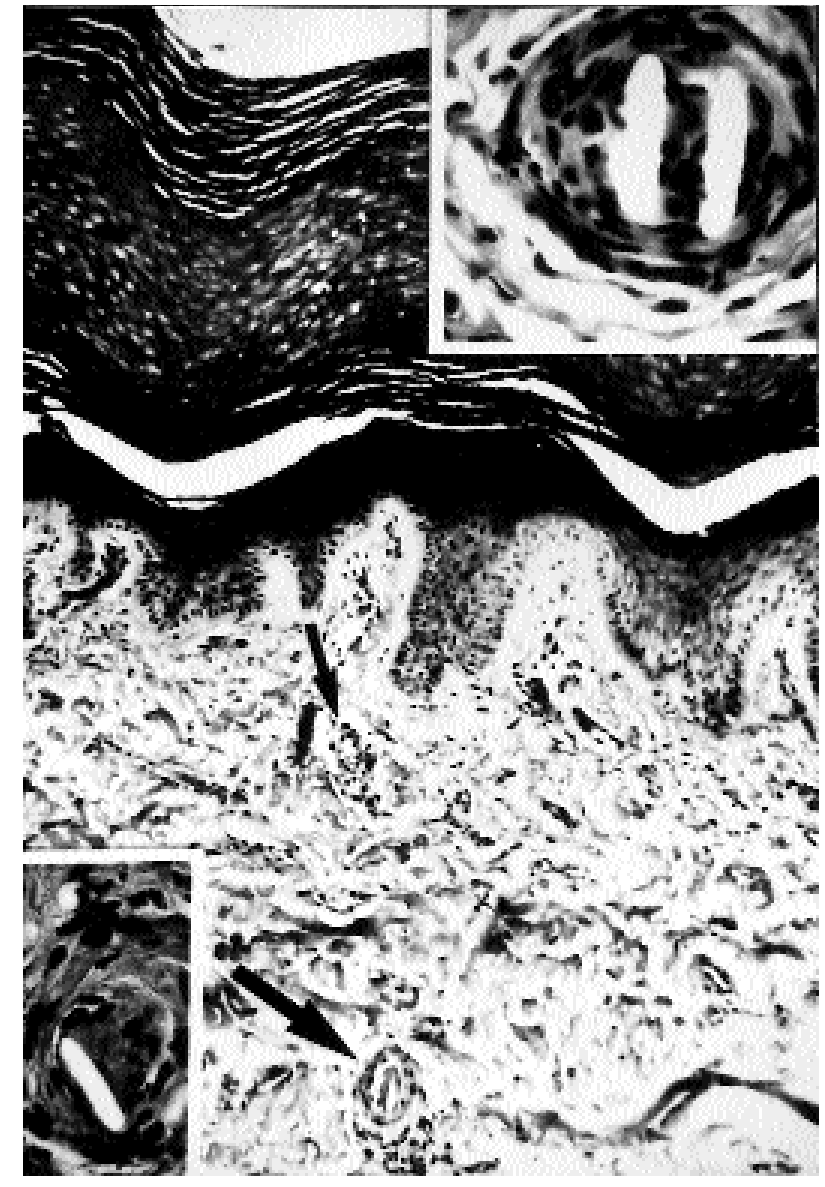

Fig. 2. Biopsia cutánea plantar. A nivel de la dermis media y profunda (flechas) existen estructura vasculares de tipo arteriolar que muestran en su luz formaciones aciculares enclavadas, a modo de hendiduras 0 siluetas ópticamente vacías (recuadros superior e inferior), correspondientes a cristales de colesterol, en visión negativa, que aparecen rodeados por elementos multinucleados gigantes. (HE, 250x).

La evolución del enfermo fue satisfactoria, sin afectación de la función renal y con desaparición del livedo reticularis y persistencia de la coloración violácea de los dedos. Se siguió un tratamiento sintomático (con analgesia y su tratamiento de la cardiopatía isquémica) y fue dado de alta siguiéndose su evolución en consultas externas.

\section{DISCUSIÓN}

La enfermedad por embolias de cristales de colesterol (EECC) es una entidad poco frecuente (según algunas series se detecta en $2-8 \%$ de las autopsias $)(1,2)$ y se presenta en pacientes varones $(78 \%)$, de edad avanzada $>65$ años (siendo excepcional su aparición por debajo de los 50 años) y con enfermedad aterosclerótica y/o factores de riesgo cardiovasculares.

El primero en describirla fue Dahlerup y Fenger (2) en su artículo sobre la autopsia del escultor Thorwaldsen, pero este logro se le atribuye a Panum en 1862 (3). La fisiopatología del cuadro se explica por la producción de una diseminación de cristales de colesterol a la circulación general desde placas de ateroma ulceradas de la aorta, que según al nivel donde estén localizadas producen la afectación de diferentes territorios vasculares. Así, si la emigración se produce a nivel de aorta abdominal se afectan las 
arterias renales, digestivas y de miembros inferiores, si es a nivel del cayado de la aorta puede haber afectación miocárdica, ocular, neurológica. Todo ello da lugar a un síndrome con manifestaciones clínicas y biológicas que corresponde con una enfermedad inflamatoria, sistémica, debido a que en el lugar de impactación del microémbolo de colesterol (100-200 micro-m de diámetro) a nivel de arteriolas de pequeño tamaño, tiene lugar una reacción a cuerpo extraño con aparición de granulomas con macrófagos, células gigantes y eosinófilos y una activación del sistema del complemento con agregación de PMN, liberación de radicales libres tóxicos en las células endoteliales que da lugar a la aparición de una hipocomplementemia transitoria por consumo y eosinofília $(4,5)$. Este cuadro se produce de forma espontánea más frecuentemente pero entre los factores desencadenantes se asocia la presencia de un factor mecánico directo: Exploración endovascular (44$60 \%$ ), cirugía cardiaca (9-18\%). Existe un grupo (62\%) que la clínica aparece en la primera semana tras la manipulación arterial y un segundo grupo (38\%) que aparece entre el décimo día y tercer mes $(6,7)$. Se han descrito casos de aparición de EECC con el tratamiento anticuagulante con antagonistas de la vitamina $\mathrm{K}$ y con menos frecuencia con heparina (8). El mecanismo de producción es discutido y se cree que es debido a la desestabilación del trombo arterial sobre las placas ulceradas del ateroma que da lugar a la diseminación de los cristales. Algunos autores piensan que el efecto es más coadyuvante y que depende de un factor mecánico casual previo. Asimismo existen casos con el tratamiento fibrinilítico (9). El plazo entre el uso de fibrinolíticos y aparición de síntomas oscila entre 1 hora y 28 días.

La descripción anatomopatológica de la EECC es característica y se basa en la identificación de los cristales de colesterol en el interior de pequeños vasos. Estos cristales pueden ser visualizados en visión negativa tras una fijación convencional en formaldehido o líquido de Bouin, si bien pueden identificarse con tinciones especificas (Schultz-Lieberman o Rojo Sudan III) utilizando cortes por congelación. Los cristales de colesterol por otra parte presentan birrefringencia a la luz polarizada. El aspecto morfológico de los cristales revela imágenes aciculares de contorno biconvexo, pudiéndose observar reacción gigantocelular tipo cuerpo extraño conformando pequeños granulomas $(10,18)$, a menudo asociados a fenómenos de proliferación subintimal o incluso en casos evolucionados a fenómenos de vasculitis.

Las manifestaciones clínicas. Son muy variadas e inespecíficas. Depende de la localización anatómica de los émbolos de colesterol, del grado y extensión de la afectación orgánica. Puede dar formas localizadas y formas generalizadas. Da signos inespecíficos (fiebre, mialgias, cefaleas) o puede simular una enfermedad sistémica con síndrome inflamatorio con manifestaciones biológicas e inmunológicas que hacen difícil el diagnóstico $(4,5)$.

La forma clínica más frecuente y aparente es la manifestación cutánea. La distribución suele ser simétrica y de predominio en MMII. Suele ir precedido o acompañado de dolor brusco y continuado, con zonas purpúricas, violáceas a nivel de dedos de los pies (Blue toe Sindrome) (13) de forma heterogénea que puede llegar a la necrosis. La forma más frecuente es la aparición de una livedo reticularis $(36.4 \%)$ en forma de entramado eritemato-violáceo en MMII e hipogastrio. Aparece una gangrena (27\%), cianosis (22\%), lesiones ulcerativas, nódulos (por lesión infiltrativa que hace difícil el diagnóstico diferencial con la Panarteritis Nudosa) (14). Estas manifestaciones son fluctuantes en el tiempo, variando de una semana a otra en extensión e intensidad con recrudescencias de las mismas.
Las formas más graves y preocupantes son las manifestaciones viscerales. Dentro de ellas las más frecuentes son los microémbolos a las arterias renales. EL signo más constante es la aparición de una insuficiencia renal fluctuante de forma rápidamente progresiva (forma de inicio en más de la mitad de los pacientes) que se produce de 2 a 6 semanas después de una angiografía. La evolución de esta insuficiencia renal puede ser hacia una IR terminal o recuperación tras varias semanas de hemodiálisis. Se asocia con mucha frecuencia a la aparición de una hipertensión arterial rebelde al tratamiento con los antihipertensivos habituales $(15,24)$. La afectación a nivel de arterias digestivas da lugar a manifestaciones variadas como dolor abdominal inespecífico; perforaciones isquémicas del estómago, intestino delgado o grueso; hemorragias digestivas. El pancreas puede afectarse dando una pancreatitis aguda. La afectación del bazo con infartos esplénicos es frecuente pero ordinariamente sin traducción clínica. Se han descrito colecistitis agudas (16). Las manifestaciones más raras son las que se producen si el émbolo parte del cayado aórtico. Puede dar lugar a un embolismo coronario con IMA; Las manifestaciones neurológicas son tipo accidente cerebro-vascular (con embolismo desde una arteria carótida o vertebral) (24), mielomalacia por afectación de arterias espinales o neuropatías periféricas que son excepcionales. Se comprende peor las manifestaciones oculares provocadas por embolias retinianas en forma de amaurosis y con más frecuencia asintomáticas, de ahí el interés del examen del fondo de ojo (10\%) (17).

Existen también manifestaciones viscerales cuyo origen es inmunológico: pericarditis, glomerulonefritis necrotizantes, neuropatías periféricas (de instauración progresiva y simétrica), manifestaciones neumológicas (infiltrados intesticiales de las bases, derrame pleural eosinófilo, hemorragia intraalveolar) $(4,5)$.

Las manifestaciones biológicas. En el contexto de este síndrome hay una alteración de datos del laboratorio comunes a toda enfermedad inflamatoria sistémica con aumento de VSG (96\%), sobre todo si existe afectación de MMII, con hipocomplementemia transitoria y eosinofilia variable $(6,18 \%)$. Puede existir anemia y leucocitosis (en casi la mitad) y leve trombopenia. Existe aumento de PCR, aumento de transaminasas, de la CPK (creatin-fosfoquinasa) en caso de afectación muscular. Lo más significativo es la afectación de la función renal con aumento de la creatinemia (82\%), de la urea y proteinuria (53\%) con microhematuria. Esta afectación es frecuente pero en diferente grado y marca el pronóstico.

En algunos casos pueden existir anticuerpos antinucleares (que dificultan el diagnóstico diferencial con las conectivopatías), pero la presencia de esta anomalía se da en sujetos normales de más de 20 años en una proporción que varía entre 12,2 y $23,9 \%$, lo que confiere poco valor a esta constatación. Los lípidos en sangre suelen ser normales o levemente aumentados.

El diagnóstico diferencial suele ser difícil con las diferentes conectivopatías, sobre todo la pan que se puede presentar como un cuadro idéntico con livedo, nódulos cutáneos y manifestaciones sistémicas y específicas como eosinofilia y aumento de la VSG (25). Otras vasculitis como LES, crioglobulinemias, enfermedad de Burguer, síndrome de Seldon y cuadros procoagulantes pueden también ser difíciles de diferenciar $(18,19)$.

El diagnóstico requiere un alto grado de sospecha debido a la similitud con estos cuadros y las manifestaciones clínicas tan variadas e inespecíficas. Se sospecha por los antecedentes de manipulación vascular, factor de riesgo ateroembólico. Se 
puede evidenciar en al fondo de ojo pequeñas masas amarillentas, brillantes, a nivel de bifurcaciones arteriales a distancia de la papila. La confirmación se basa en la visualización de cristales de colesterol a nivel de una biopsia cutánea en zona de cruce de malla de livedo reticular cuya sensibilidad depende del área elegida y sobre todo de la profundidad (9-35\%) pudiendo ser necesarias múltiples y seriadas $(4,5,20)$. La biopsia muscular debe ser sistemática si la cutánea ha sido negativa, si no existen lesiones cutáneas, si existe riesgo de necrosis,. El rendimiento sería superior y se recomienda practicar a nivel del cuadriceps o triceps sural. La biopsia medular practicada a nivel del hueso iliaco es suficientemente simple y rentable para que haya sido propuesta de manera sistemática por diferentes autores. En la biopsia renal los cristales de colesterol se encuentran en los diferentes elementos de la circulación cortical renal $(11,12)$.

Pronóstico: La EECC presenta una elevada mortalidad y escasa respuesta al tratamiento. En la serie de 334 pacientes a la que hace referencia Suc en 1989 la tasa de mortalidad se eleva al $75 \%$. El estudio de las observaciones publicadas demuestra un $54 \%$ de mortalidad. Las causas de mortalidad son: Fracaso cardiaco 36.6\%, fracaso multivisceral 17\%, afectación digestiva grave $17 \%$, AVC 2,4\%, insuficiencia renal $2,4 \%$. Esta mortalidad depende del territorio y afectación de órganos nobles. La morbilidad en caso de supervivencia es elevada precisando depuración extrarrenal un $27 \%$, amputación de segmentos de miembros un 13\%, laparotomía exploradora o resecciones intestinales un 10,4\%.

Tratamiento: No existe tratamiento curativo de las lesio- nes isquémicas. En teoría el tratamiento etiológico sería erradicar la lesión embolígena: Resección del aneurisma aórtico, endoarterioectomía, prótesis de aorta abdominal arteroesclerótica. En la clínica diaria el tratamiento es sintomático y no altera la evolución natural de la enfermedad. Si parece existir un acuerdo entre los diferentes autores de la contraindicación absoluta al uso de anticuagulantes orales y de heparina por reagudización del cuadro. Se usan antiagregantes plaquetarios, vasodilatadores, simpatectomía lumbar, etc. También antiinflamatorios tipo colchicina $(4,5)$. En la literatura se describen casos en los que la corticoterapia va acompañada de mejoría clínica y biológica, pero otros autores no señalan ninguna mejoría con su uso. Los protocolos terapéuticos no son homogéneos y la dosis varía entre $0,5-1 \mathrm{mg} / \mathrm{kg} /$ día según diferentes publicaciones. Se debería posponer la terapia corticoidea en casos de isquemia de extremidades mal controladas por otras medidas terapéuticas, en casos de afectación multivisceral con alteración del estado general y síndrome inflamatorio importante, en caso de manifestaciones clínicas no directamente relacionadas con la obliteración arterial por cristales de colesterol (pericarditis, multineuritis, hemorragia alveolar) $(21,22)$. Se debe tratar las complicaciones tipo insuficiencia renal con hemodiálisis y/o diálisis peritoneal. Utilización de antihipertensivos, ect. Algunos autores han utilizado lovastatina con mejoría de la lesión de ateroma (23). En definitiva, hay que ir tratando de forma sintomática las diferentes complicaciones, manteniendo una actitud expectante vigilando la evolución del cuadro, que se altera poco con las diferentes terapias utilizadas.

\section{Bibliografía}

1. Moolenaar W, Lamers CBHW. Cholesterol Crystal Embolization in the Netherlands. Arch Intern Med 1996; 156: 653-57.

2. Dahlerup, Fenger. Obduktionsbericht. Ugeskr Laeger 1944; 1: 215.

3. Panum PL. Experimentelle Brealtrage zur lehre von der embolie. Virchows Arch Pathol Anat Physiol 1862; 25: 308-10.

4. Claudel JP, Ninet J, Coppere B. Embolies de cholestérol dans les membres inférieur. Rev-Prat (Paris) 1995; 45: 56-61.

5. Blétry O, et al. Les embolies de cholesterol. Rev-Prat (Paris) 1986; 36:23.

6. Colt HG, Begg RJ, Suporito JJ, Cooper WM, Shapiro AP. Cholesterol emboli after cardiac catheterization: eight cases and review of the literature. Medicine 1988; 347: 1641.

7. Barrabés Riu JA, Tolosa Vilella C, Ordi Ros J, Premanyer Miranda G, Salas Caudevila A. Embolismo de colesterol tras angioplastia coronaria. Presentación de un caso. Rev Esp Cardiol 1991; 44: 411-413.

8. López Pardo F, González Barrero A, Serrano Castro V, Andreu Alvarez J, Navarrete Ortega M, Burgués Cornejo J. Embolismo de colesterol durante el tratamiento con heparina. Rev Esp Cardiol 1993; 46: 755-764.

9. Ridker PM, Michel T. Streptokinase therapy and cholesterol embolization. Am J Med 1989; 87: 357-358.

10. Flory CM. Arterial occlusion produced by embolism from eroded atheromatous paque. Am J Pathol 1945; 21: 549-565.

11. Gore I, Colins DP. Spontaneus Atheromatous Embolization. Am J Clin Path 1960; 33: 416-426.

12. Anderson WR. Necrotizing angeitis associated with embolization of cholesterol. Am J Clin Path 1964; 43: 65-71.

13. Ramdas G, Pai RG, Heywood JT. Atheroembolism (Images in clinical medicine). N Engl J Med 1995; 333 (13): 852.

14. Vargas E, Puig L, Curell R, De Moradas JM. Embolización por cristales de colesterol. Piel 1992; 7: 186-188.

15. De la Casa Monje R, Martínez Ara J, Cardenal A, Picazo ML. Embolismo renal por cristales de colesterol: Aparición tardía de la insuficiencia renal. [carta]. Med Clin (Barc) 1992; 98: 597-598.

16. MoolenaarW, Lamers CBHW. Cholesterol crystal embolization to the alimentary tract. Gut 1996; 38: 196-200.

17. Bradley M. Spontaneus Atheroembolism (Images in clinical medicine) N Engl J Med 1995; 332 (15): 998.

18. Rhodes JM. Embolismo de cristales de colesterol: un "nuevo" diagnóstico importante para el generalista [carta]. The Lancet 1996; 347 : 1641.

19. Fuerte MJ, Bielsa I, Ribera M, Pellicer I, Ferrándiz C. Enfermedad por émbolos de colesterol. Rev Clin Esp 1994; 194: 23-25.

20. Macías MD, Guerediaga J, Velasco J, Lagunas IL. La biopsia cutánea en la nefropatía ateroembólica. Med Clin (Barc) 1990; 95: 793-794.

21. Hachulla B, Devulder B. Traitement médical des embolies de cristaux de cholestérol. Presse Médicale 1991; 20: 215-219.

22. Robert M, Applebaum MD, Itzhak MD. Evaluation and management of cholesterol embolization and the blue toe syndrome. Curr Op Cardiol 1996; 11: 533-542.

23. Cabili S, Hochman I, Goor Y. Revelsal of gangrenous lesions in the blue toe syndrome with lovastatin, a case report. Angiology 1993; 44: 821-825.

24. García Cors M, Montull S, Blanco M, López Alonso G, Castellón E, Pasquau J, et al. Ateroembolismo de colesterol: Accidente cerebrovascular agudo e insuficiencia renal aguda como forma de presentación. An Med Interna (Madrid) 1986; 3: 281-284.

25. Susano R, Caminal L, Gómez C, Fernandez J, De Quirós B. Enfermedad por embolismos múltiples de colesterol que se presentó como una panarteritis nodosa. [carta]. An Med Interna (Madrid) 1994; 11: 619.

26. Cruz Vicente JM. Enfermedad ateroembólica (embolismo por cristales de colesterol). An Med Interna (Madrid) 1997; 14: 257-262. 